\title{
Elastic 'tethers' connect separating anaphase chromosomes in a broad range of animal cells.
}

Arthur Forer ${ }^{1 *}$, Michelle L. Duquette ${ }^{2}$, Leocadia V. Paliulis ${ }^{3}$, E. Fegaras ${ }^{1}$, M. Ono ${ }^{2}$, D. Preece ${ }^{2}$, Michael W. Berns ${ }^{2,4}$

1: Biology Department, York University, North York, Ontario, Canada M3J 1P3

2: Department of Bioengineering and Institute for Engineering in Medicine, University of California, San Diego 92093

3: Biology Department, Bucknell University, Lewisburg, PA 17837

4: Beckman Laser Institute and Department of Biomedical Engineering, University of California, Irvine, CA 92617

*Correspondence to: Arthur Forer, Biology Department, York University, North York, Ontario M3J 1P3, Canada. aforer@yorku.ca

Running Head: Backward forces on anaphase chromosomes 


\begin{abstract}
We describe the general occurrence in animal cells of elastic components ("tethers") that connect individual chromosomes moving to opposite poles during anaphase. Tethers, originally described in crane-fly spermatocytes, produce force on chromosome arms opposite to the direction the anaphase chromosomes move. In crane-fly spermatocytes tethers function to coordinate movements between chromosomes. Their presence in a broad range of cells suggests that they may be important in coordinating movements between chromosomes to ensure normal segregation. Tethers are previously unrecognised force-producing components of general mitotic mechanisms and need to be accounted for in general models of mitosis in terms of forces on chromosomes and in terms of what their roles might be, possibly in coordinating chromosome movements during mitosis.
\end{abstract}

\title{
INTRODUCTION
}

This article describes the presence of elastic connections between separating anaphase chromosomes in cells from a broad range of animals, from flatworms to humans, suggesting that such connections are universal. Elastic connections between chromosomes moving to opposite poles represent previously unrecognised forces on anaphase chromosomes, acting in opposite direction to the forces moving chromosomes to the poles. While the composition and function of these connections are unknown, their universal presence has broad implications to how chromosome movements in anaphase are coordinated and controlled.

Elastic physical connections between arms of separating chromosomes were originally demonstrated in anaphase crane-fly spermatocytes by LaFountain et al. (2002a), who called them "tethers". Tethers were identified operationally: fragments cut from ends of arms moved 'backwards' to the partner telomeres that were moving to the opposite pole. They moved with speeds considerably greater than the chromosomes while the severed chromosome continued moving to its original pole. Further experiments by LaFountain et al. (2002a) indicated that these 'backwards' movements of arm fragments were not due to forces from motors associated with spindle microtubules, and pointed strongly to forces arising from elastic connections between separating telomeres, as follows.

When backwards moving arm fragments were cut into two pieces, the sub-fragment with the telomere kept moving but the other one did not: it remained motionless. Because the fragment without the telomere did not move, this indicates that arm-fragment movement is not due to generalised spindle transport forces acting on arms in general. Rather it points toward something acting on the telomere. In other experiments telomeres were ablated directly: the arm fragment stopped moving after ablation of either the telomere of the arm-fragment or the telomere of the partner (intact) chromosome. Thus the forces for movement are applied specifically at the telomere, both telomeres need to be intact, and the forces seem to arise from elastic connections 
('tethers') that extend between separating telomeres. That the movement of fragments is not due to forces from microtubule motors is further shown in the anaphase behaviour of other arm fragments. Acentric arm fragments were produced from metaphase chromosomes. Those that remained in the cell during anaphase (LaFountain et al., 2001, 2002b) moved poleward to the nearest pole at about the same speed as normal anaphase chromosomes (LaFountain et al., 2001). These observations confirm earlier experiments in a variety of cells that show the same thing, that both granules pushed into the spindle and acentric chromosome fragments are transported to the nearest pole at speeds about the same as normal anaphase chromosome movements (Ostergren et al., 1960; Bajer, 1967; Nicklas and Koch, 1972). This is in contradistinction to arm fragments produced in anaphase which move away from the nearest pole (across the equator to the partner telomere) at much faster speeds than chromosome movements. Thus, the movements of arm fragments across the equator to their partner telomeres are not due to forces from microtubules in the spindle but to some component(s) producing force in the opposite direction, 'tethers', elastic connections of unknown composition.

Tethers were identified operationally in anaphase meiosis-II cells (LaFountain et al., 2002a) as well as meiosis-I cells, so they are not restricted to reduction divisions. Tethers do not connect all arms of separating chromosomes. In meiosis-I, only two of the four arms of each separating half-bivalent pair are connected by tethers (LaFountain et al., 2002a): the arm fragments with tethers move backwards but the other two arm fragments do not, further indicating that the backward movements are not due to generalised forces from microtubules acting on chromosome arms but to specific connections between partner telomeres. The tethers seem to become less elastic as anaphase progresses. Arm fragments produced in early anaphase move fast and reach the telomere of the separating chromosome. Those produced later move across the equator at slower speeds and stop before reaching the partner telomere. Those produced yet later move somewhat toward the partner telomere but not across the equator, or do not move at all. LaFountain et al. (2002a) interpreted this behaviour to mean that as anaphase proceeds tethers lose elasticity, as indicated by fragments slowing down, and that eventually tethers are no longer attached to telomeres, as indicated by absence of motion.

In this report we identify and characterise tethers in PtK (marsupial tissue culture) cells, and identify tethers in cells ranging from flatworms to humans, namely from a turbellarian flatworm, two classes of insects, two distinct lineages of arachnids, a marsupial, and humans. Thus, elastic tethers connect separating anaphase chromosomes in animal cells in general, if not universally.

\section{MATERIALS AND METHODS}

Cells: Asynchronous or rapidly proliferating PtK2 and U2OS cells were maintained in Dulbecco's modified Eagle's medium (Invitrogen, Carlsbad, CA) supplemented with 10\% bovine calf serum, L-glutamine, and sodium pyruvate) at $37^{\circ} \mathrm{C}$ and $5 \% \mathrm{CO} 2$.

Adult Pholcus phalangioides (Fuesslin) (cellar spider) males were collected from a field

site near Bucknell University, Lewisburg PA. Adult Latrodectus mactans (Fabricius) (black 
widow spider) males were obtained from Spider Pharm Inc. (Yarnell, AZ). The abdomens of spiders were cut away from the cephalothorax. Testes were removed from the abdomen and placed in a culture chamber as described in (Ault et al., 2017) Testes contents were spread under a layer of Kel-F oil (Ohio Valley Specialty Chemical, Marietta, $\mathrm{OH}$ ).

Juvenile Acheta domesticus (Linnaeus) male crickets were purchased from PetCo (Clairemont, San Diego, California). Testes were removed through a small incision cut directly behind the wing buds on the dorsal side of the cricket abdomen. Testes were placed directly into Kel-F oil and testes contents were spread under a layer of Kel-F oil.

Crane flies, Nephrotoma suturalis (Loew), were from a laboratory culture (Forer, 1982) and spermatocytes were put into a fibrin clot and immersed in Ringers solution as described in Forer and Pickett-Heaps (2005).

The turbellarian flatworms, Mesostoma ehrenbergii, were from laboratory cultures (Hoang et al., 2013). Spermatocytes were removed from animals, placed in fibrin clots, and immersed in Ringers solution as described in Hoang et al. (2013) and Ferraro-Gideon et al. (2014). The highly unusual chromosome movements in these cells are described in detail in FerraroGideon et al. (2014).

Experimental methods: The viewing, irradiation, and analysis methods are described detail in Forer et al. (2013). In brief, cells were observed using phase-contrast microscopy (using a Zeiss Plan-Neofluar 63X lens, NA 1.4) in a microscope fitted for laser microbeam surgery (Harsono et al. 2012; Shi et al. 2012). Regions of interest were irradiated using a 100-fs pulsed laser (Mai Tai, Newport Co., Irvine, CA, USA) emitting 740nm wavelength light. Cells were observed at room temperature, including some of the PtK cells, but for all experiments on U2OS cells and most experiments using PtK cells, the cells were placed on a heated stage maintained at $36.5^{\circ} \mathrm{C}$. Images recorded every 2 or 3 seconds were cropped, stamped with date and time, and converted into bmp files using IrfanView freeware. The bmp files were assembled into avi files using VirtualDub freeware. We analysed the results of the experiments by visual observation and by obtaining distance versus time graphs, as described in Forer et al. (2013), using an in-house program, WinImage (Wong and Forer, 2003). The graphical data on chromosome arm shortening were only from cuts that caused arms to shorten, except for Figures 10 and 12, which included all irradiations. Montages were assembled from individual images using Photoshop. The images were adjusted only for image contrast and brightness levels.

\section{RESULTS}

\section{Tethers in PtK2 cells:}

Tethers connect separating anaphase chromosomes in PtK2 cells. When chromosome arms in anaphase cells are cut using a laser microbeam, the resultant arm fragments move away from the arm stub, toward their partner telomeres, either (a) reaching them, or (b) stopping across the equator before reaching them, or (c) stopping before crossing the equator (Fig 1). The velocities 
of arm fragments were faster than anaphase chromosomes in the same cells (TABLE 1), with fragment velocities as high as $6.2 \mu \mathrm{m} / \mathrm{min}$.

Arm fragment movements are due to elastic connections between telomeres, because cutting the region between the two telomeres causes the arm fragment to stop moving toward the opposite chromosome (Figure 2). [This was our operational definition of cutting tethers.] When tethers were cut, some arm fragments reversed direction and moved back towards the arm stub (Figure 3), as reported for fragments in crane-fly spermatocytes (Sheykhani et al., 2017), presumably because the initial arm-severing cut was incomplete and elastic chromosome components remained in the cut region.

Tension from tethers stretches anaphase crane-fly spermatocyte chromosome arms backward, toward the separating partner telomeres (LaFountain et al., 2002a). Stretched arms shorten when their tethers are cut (Sheykhani et al., 2017). Thus we cut PtK-cell tethers themselves, to test whether their chromosomes were stretched. After cutting their tethers, arms shortened (Figure 4). In those cells in which we were able to see both kinetochore and telomere sufficiently clearly to measure arm lengths, the arms shortened to about $89 \%$ of their initial value $(0.89 \pm 0.025 \mathrm{sd}, \mathrm{N}=31)$, over a period of up to a minute, independent of the initial length of the chromosome arm (Figure 5). The stretch appears to be uniform along the entire length of the chromosome arm, because, independent of the initial length of the stub, the arm stubs shortened to about the same extent $(0.85 \pm 0.06 \mathrm{sd}, \mathrm{N}=10)$ as entire arms (Figure 6).

Not all arms shortened when we attempted to cut their tethers. This may be because we did not actually cut them; we have no visible marker of tethers, so it is possible that sometimes they were not cut.

Not all arm fragments moved backwards toward the partner telomere. Lack of movement might be because not all arms are connected to their partners with tethers. For example, in some cells only one or two arm fragments moved backwards toward the other telomeres while others cut at the same time and same tether length (as measured by inter-telomere distance) did not (Figure 7, and supplemental video). It is unclear which arms are connected by tethers and which not, though it may be that, as in crane-fly spermatocytes, there are tethers between only one of the two arms of each separating chromosome. Another possibility why some arm fragments did not move might be that tethers in PtK cells become less elastic as anaphase progresses. To test whether longer tethers are less elastic than shorter ones we compared arm fragment velocities with arm shortening after cutting tethers.

Arm fragment movement varied with tether length. The longer the tethers, (a) the slower were the velocities of arm fragments (Figure 8), (b) the less likely the arm fragments were to reach the partner telomere or to cross the equator (Figure 9), and (c) the less likely that arm fragments would move at all (Figure 10). On the other hand, the amounts the arms shortened after cutting tethers did not change with tether length: at all tether lengths the arms shortened on average to $89 \%$ of their initial length (Figure 11). Thus while arm fragment velocities were slower as anaphase progressed, the tension that the arms were under did not change. This suggests that tethers are present between separating telomeres at all tether lengths, at least to the maximum we studied 
$(9.6 \mu \mathrm{m})$, and that their elasticity is reduced as they become longer. As further indication that this is the case, arms shortened after cutting tethers at tether lengths at which fragments did not move: at tether lengths longer than $7 \mu \mathrm{m}$, none of 17 arms fragments moved (Figure 10) yet at those same lengths $(>7 \mu \mathrm{m})$ arms shortened after 6 out of 8 cuts of tethers (Figure 12). Thus tethers are present and produce a constant tension on the arms throughout anaphase, but the elasticity of the tethers decreases as the tethers elongate.

We did not detect any change in poleward movement of anaphase chromosomes after their tethers were cut, so the force from the tethers that stretches chromosome arms is much smaller than the force moving the chromosome to the pole.

\section{Tethers in crane-fly spermatocytes.}

Tether properties described in in previous work on crane-fly spermatocytes (LaFountain et al., 2002a; Sheykhani et al., 2017) are the same as those we found in PtK cells. Some issues had not been determined for crane-fly spermatocytes, however, namely, whether the tethers lose elasticity as they get longer and how much the tethers stretch the chromosome arms to which they are attached. We studied crane-fly spermatocytes to look at these particular issues.

In crane-fly spermatocytes, chromosomes shortened on average to $89 \%$ of their initial length, similar to that in PtK cells (TABLE 2). Arms shortened in late anaphase when their tethers were cut, up to tether lengths of at least $15 \mu \mathrm{m}$ (the longest we have studied); at these tether lengths arm fragments do not move, or move very slightly (LaFountain et al., 2002a). Thus our data suggest that, similar to PtK cells, tethers in crane-fly spermatocytes are present throughout anaphase but lose their elasticity as anaphase progresses.

\section{Tethers in other cells.}

We have identified tethers in other cell types as well, from flatworms to humans. Arm fragments produced in early anaphase moved across the equator to the partner telomeres in spermatocytes from the turbellarian flatworm Mesostoma; in spermatocytes from an orthopteran insect, the house cricket Acheta domesticus, in both meiosis-I and meiosis-II; in spermatocytes from two arachnids from distinct lineages, cellar spiders (Pholcus) and black widow spiders (Latrodectus); and in human osteosarcoma cancer cells, U2OS (Figure 13). We have seen at least 3 examples of rapid arm fragment movements in each of the cells studied. Those whose speeds we have been able to measure are presented in TABLE 3. In addition, we have verified several other characteristic of tethers in these cells: in particular, in all of the cells not all arm fragments move

toward their partner; in flatworm spermatocytes, cricket spermatocytes and human cells tethers stretch arms; and in cricket spermatocytes cutting tethers stops the movements of arm fragments.

\section{DISCUSSION}

A main conclusion from our experiments is that there are mechanical connections (tethers) between separating anaphase chromosomes in a broad range of cells: flatworm spermatocytes, crane-fly spermatocytes (meiosis-I and -II), cricket spermatocytes (meiosis-I and II), cellar spider 
spermatocytes (meiosis-I), black widow spider spermatocytes (meiosis-I), marsupial (PtK) cells, and human U2OS (osteosarcoma) cells. Detailed characterisation of tethers in PtK cells and cranefly spermatocytes indicates that tethers are elastic, but that they lose elasticity as they become longer: the arm fragments move slower and slower toward the opposite telomere, and eventually do not cross the equator or do not move. Severing tethers releases the connections between separating arms which causes the arms to shorten. Arms shorten at all tether lengths, whereas arm fragments do not move at longer tether lengths. Thus separating chromosomes are connected by tethers throughout anaphase but the longer the tether the less elastic it is. The force exerted by tethers on separating chromosomes stretches the chromosome arms by around 12\% (100/89) regardless of tether length; the stretch seems to be throughout the length of the chromosome arms since the chromosome stubs (that remain after the arm is severed) also contract to the same extent as the unsevered arm, at least in PtK cells, in which the arm stubs are long enough to measure length changes. The force exerted by tethers is enough to stretch chromosome arms but is smaller than that exerted on the kinetochore toward the poles since chromosomes do not speed up when tethers are cut (this paper, also LaFountain et al., 2002a; Sheykhani et al., 2017).

Because tethers are found in cells from widely separate organisms, from flatworms to humans, it seems likely that they are present in most cell types, and perhaps are even present universally in cells, at least those with localised kinetochores. It seems likely that tethers between partner chromosomes are responsible for the backwards movements observed after cutting individual anaphase spindle fibres in PtK cells (Elting et al., 2014), which have tethers (this paper), and, by extension, in grasshopper spermatocytes (Chen and Zhang, 2004) and newt fibroblasts (Spurck et al., 1997), in neither of which have tethers been demonstrated. We might also speculate that backwards movements seen after cutting across entire half-spindles in anaphase Haemanthus cells (Bajer, 1972) are due to tethers, and backwards movements after spindle poles are irradiated in silkworm meiosis-I (Nakanishi et al., 1965) are due to tethers.

Our purpose in the experiments presented herein was to test whether tethers were present in a variety of cells, to generalise their presence. We envision that subsequent experiments will deal with the question, what are tethers composed of? We did not pursue this question, and we have no data regarding their composition. But perhaps brief discussion is worthwhile.

It is difficult to get a handle on tethers if one cannot identify them so we would like to be able to see them in living cells, or at least in stained cells. Since cutting between partner telomeres in PtK cells caused chromosome arms to shorten $75 \%$ of the time (Figure 12), we expect tethers to extend between separating telomeres in at least $75 \%$ of the separating chromosomes, and most likely $100 \%$. In crane-fly spermatocytes tethers were identified operationally for each pair of separating chromosomes, but only for half the arms (La Fountain, 2002b). In trying to identify tethers morphologically we therefore expect to see them between all separating chromosomes, though not necessarily between all separating arms. Connections between separating telomeres in anaphase are not visualised by several usual staining methods (discussed in Sheykhani et al., 2017) and no connections between telomeres were seen in PtK cells using standard electron microscopy sectioning methods (Roos, 1973). However, connections between separating telomeres were seen electron microscopically in crane-fly spermatocytes by Fuge (1978), who reported that in 
fortuitous sections of two crane-fly spermatocytes he identified connections between telomeres in three of the 6 bivalent pairs; in one of the pairs there were connections between two of the 4 pairs of arms, similar to the results from cutting arms in living cells (LaFountain et al., 2002a; Sheykhani et al., 2017). Connections between separating chromosomes also were seen electron microscopically in meiosis-I of cockroaches (Krishan and Buck, 1965). Given the difficulty of spotting such elements using the electron microscope (Fuge, 1978), it is tempting to think that these components correspond to tethers, but one does not know how regularly this occurs - i.e, whether all chromosomes are connected this way. Without that correlation one does not know, without further experimentation, whether these images are of tethers. Some bridges also have been seen using light microscopy: Feulgen-positive chromatin bridges that are composed of chromatin and are visible using standard light microscopy after staining for DNA, and ultra-fine DNA bridges that do not stain for DNA but are identified by staining for associated proteins BLM, topoisomerase-III $\alpha$, or PICH (Chan et al., 2007). Neither of these DNA bridges fits the criteria for tethers, however. Chromatin bridges (sensu Schrader, 1953, pp 45-46) are present only rarely in healthy normal cells, and when present generally retard or stop movements. Ultra-fine (DNA) bridges, studied primarily in human tissue culture cells, also do not fit the criteria: in different reports only $20-80 \%$ of normal anaphases have 1 or more ultra-fine bridges (Chan et al., 2007; Barefield et al., 2012; Gemble et al., 2015), yet tethers are present in all cells. Of those anaphase cells that do have ultra-fine bridges, in different reports there are less than 8 bridges per cell (Chan et al., 2007), or 2 or more bridges in only 50\% of the cells (Barefield et al., 2012), or more than 3 bridges per cell in only $10-15 \%$ of the cells (Gemble et al., 2015), considerably fewer than the 46 pairs of chromosomes, yet tethers are associated with at least $75 \%$ of the chromosomes, if not all. Further, of the bridges studied, most terminate in centromeric DNA or 'fragile sites', not telomeres (Chan et al., 2007, 2009; Barefield et al., 2012; Nielsen et al., 2015). Those extending from telomeres at one or both ends of the bridge were only $15 \%$ of the identified ultra-fine bridges (Barefield et al., 2012). Thus, the ultrafine DNA bridges identified by these staining methods cannot be components of tethers since not all cells have them and there are far too few per cell that extend between telomeres.

We are aware of only one other description of components extending between separating anaphase chromosomes, those containing the protein titin, described as extending between separating anaphase telomeres in crane-fly spermatocytes (Fabian et al., 2007). Though titin has elastic properties consistent with it being a tether component, we do not know from the published report whether all chromosomes have them, or how often they are seen, so without further description and experimentation one cannot be certain whether they might be components of tethers.

Why do tethers extend between separating anaphase chromosomes? What is their function? One can only speculate at this time, but experiments on crane-fly spermatocytes (Sheykhani et al. 2017) indicate that tethers in crane-fly spermatocytes function to coordinate movements of separating chromosomes; this conclusion derived from studying movements that were linked when tethers were present but that became unlinked when tethers were severed. Thus one function in crane-fly spermatocytes is to modulate movements between partner chromosomes, possibly through the tension that they generate on chromosome arms. Perhaps their evolutionary function 
is to coordinate chromosome movements in general, to help ensure that chromosomes are directed to their proper poles.

How much force does a tether produce? The force is enough to stretch chromosomes by $12 \%$, but is smaller than the poleward force that acts on the kinetochore. Nonetheless, small as it may be, the forces from tethers oppose the poleward forces at kinetochores, and should be taken into account when considering the balance of forces acting on chromosomes during anaphase. We plan to measure the force that drives arm fragment motion by using an optical trap to stop the movement of arm fragments shortly after they are formed.

In summary, we have shown that tethers extend between the telomeres of separating chromosomes in a broad range of anaphase cells, both meiotic and mitotic. They exert a force that opposes the forces propelling the chromosome to the pole, enough to stretch the chromosome arm by $12 \%$. At the start of anaphase the tethers are elastic, but they lose elasticity as they elongate. Tethers have been found in the several cell types we have studied, the only ones where they have been looked for, as far as we know. From the range of species in which tethers are present, tethers probably are present in most cells, if not universally present during cell division.

ACKNOWLEDGEMENTS. Supported by grants from the Natural Sciences and Engineering Research Council of Canada (to AF) and from the Beckman Laser Institute Foundation and from the Air Force Office of Scientific Research FA9550-10-1-0538 (to MWB). LP was supported by a Scadden Fellowship from Bucknell University. None of the authors have any conflict of interest.

\section{REFERENCES}

Ault JG, Felt KD, Nedo AO, Doan RN, Andreychik CM, Paliulis LV (2017). CoSegregation of sex chromosomes in the male black widow spider Latrodectus mactans (Araneae, Theridiidae). Chromosoma doi:10.1007/s00412-017-0628-7

Bajer A (1967). Notes on ultrastructure and some properties of transport within the living mitotic spindle. J Cell Biol 33, 713-720.

Bajer A (1972). Influence of UV microbeam on spindle fine structure and anaphase chromosome movements. Chromosomes Today 3, 63-69.

Barefield C, Karlseder J (2012). The BLM helicase contributes to telomere maintenance through processing of late-replicating intermediate structures. Nucleic Acid Res 40, 7358-7367.

Chan K-L, North PS, Hickson ID (2007). BLM is required for faithful chromosome segregation and its localization defines a class of ultrafine anaphase bridges. EMBO J 26, 33973409. 
Chan KL, Palmai-Pallag T, Ying S, Hickson ID (2009). Replication stress induces sisterchromatid bridging at fragile site loci in mitosis. Nature Cell Biol 11, 753-761.

Chen W, Zhang D (2004). Kinetochore fibre dynamics outside the context of the spindle during anaphase. Nature Cell Biol 6, 227-231.

Elting MW, Hueschen CL, Udy DB, Dumont S (2014). Force on spindle microtubule minus ends moves chromosomes. J Cell Biol 206, 245-256.

Fabian L, Xia X, Venkitaramani DV, Johansen KM, Johansen J, Andrew DJ, Forer A (2007). Titin in insect spermatocyte spindle fibers associates with microtubules, actin, myosin and the matrix proteins skeletor, megator and chromator. J Cell Sci 120, 2190-2204.

Ferraro-Gideon J, Hoang C, Forer A (2014). Meiosis-I in Mesostoma ehrenbergii spermatocytes includes distance segregation and inter-polar movements of univalents, and vigorous oscillations of bivalents. Protoplasma 251,127-143

Forer A (1982). Crane fly spermatocytes and spermatids: a system for studying cytoskeletal components. In: Methods in Cell Biology, ed L.Wilson, Vol 25. New York: Academic Press, 227 252.

Forer A, Ferraro-Gideon J, Berns MW (2013). Distance segregation of sex chromosomes in crane-fly spermatocytes studied using laser microbeam irradiations. Protoplasma 250,10451055.

Forer A, Pickett-Heaps J (2005). Fibrin clots keep non-adhering living cells in place on glass for perfusion or fixation. Cell Biology International 29, 721-730

Fuge H (1978). Fine structure of anaphase bridges in meiotic chromosomes of the crane fly Pales. Chromosoma 65, 241-246.

Gemble S, Ahuja A, Buhagiar-Labarchède G, Onclercq-Delic R, Dairou J, Biard DS, Lambert S, Lopes M, Amor-Guéret (2015). PLOS Genetics DOI:10.1371/journal.pgen.1005384.

Harsono MS, Zhu Q, Shi LZ, Duquette M, Berns MW (2012). Development of a dual joystick-controlled laser trapping and cutting system for optical micromanipulation of chromosomes inside living cells. J Biophotonics. doi:10.1002/jbio.201200019

Hoang C, Ferraro-Gideon J, Gauthier K, Forer A (2013). Methods for rearing Mesostoma ehrenbergii in the laboratory for cell biology experiments, including identification of factors that influence production of different egg types. Cell Biol Int 37, 1089-1105.

Krishan A, Buck RC (1965). Ultrastructure of cell division in insect spermatogenesis. J Ultrastruct Res 13,444-458. 
LaFountain JR, Cole RW, Reider CL (2002a). Partner telomeres during anaphase in crane fly spermatocytes are connected by an elastic tether that exerts a backward force and resists poleward movement. J Cell Sci 115,1541-1549.

LaFountain JR, Cole RW, Reider CL (2002b). Polar ejection forces are operative in cranefly spermatocytes but their action is limited to the spindle periphery. Cell Motility Cytoskel 51, 16-26.

Nakanishi YH, Kato H (1965). Unusual movement of the daughter chromosome group in telophase cells following the exposure to ultraviolet microbeam irradiation. Cytologia 30, 213221.

Nicklas RB, Koch CA (1972). Chromosome manipulation. IV. Polarized motions within the spindle and models for mitosis. Chromosoma (Berl) 39, 1-26.

Nielsen CF, Huttner D, Bizard AH, Hirano S, Li T-N, Palmai-Pallag T, Bjerregaard VA, Liu Y, Nigg EA, Wang L H-C, Hickson ID (2015). PICH promotes sister chromatid disjunction and co-operates with topoisomerase II in mitosis. Nature Comm 6: 8962| DOI:10.1038/ ncomms 9962

Östergren G, Mole-Bajer J, Bajer J (1960). An interpretation of transport phenomena at mitosis. Ann NY Acad Sci 90, 381-408.

Roos P (1973). Light and electron microscopy of rat kangaroo cells in mitosis. I. Formation and breakdown of the mitotic apparatus. Chromosoma 40, 43-82.

Schrader F (1953). Mitosis. The movements of chromosomes in cell division. Columbia University Press, N.Y. Second edition.

Shi LZ, Zhu Q, Wu T, Duquette M., Gomez V, Chandsawangbhuwana, C, Harsono MS, Hyun N, Baker N, Nascimento J, You Z, Botvinick EB, Berns MW (2012). Integrated optical systems for laser nanosurgery and optical trapping to study cell structure and function. In: Current microscopy contributions to advances in science and technology, ed. A Mendez-Vilas, Formatex, Badajoz, Spain. Microscopy Book Series Number 5,685-695.

Sheykhani R, Berns M, Forer A (2017). Elastic tethers between separating anaphase chromosomes in crane-fly spermatocytes coordinate chromosome movements to the two poles. Cytoskeleton 74, 91-103.

Spurck T, Forer A, Pickett-Heaps J (1997). Ultraviolet microbeam irradiations of epithelial and spermatocyte spindles suggest that forces act on the kinetochore fibre and are not generated by its disassembly. Cell Motil Cytoskel 36, 136-148.

Wong R, Forer A (2003). 'Signalling' between chromosomes in crane-fly spermatocytes studied using ultraviolet microbeam irradiation. Chromosome Res 11, 771-786. 


\section{FIGURE LEGENDS}

Figure 1: arm fragments move across the equator to their partner telomeres in a PtK cell. Figure 1A: the white line in the second image is the laser position immediately after the arms were cut. The arm fragment indicated by the white arrow moved to the partner telomere. The other arm fragments did not. The black line in the first image represents $5 \mu \mathrm{m}$. Figure 1B: The white lines in the second and third images represent the laser positions just as and just after the arms were cut. The arm fragment indicated by the white arrow moved to the partner telomere and when it did the partner chromosome shortened, and the arm stub shortened. The other arm fragment did not move. The black line in the first image represents $5 \mu \mathrm{m}$.

Figure 2: cutting the tether stops movement of the arm fragment in a PtK cell. The white arrow indicates the arm fragment. The arm fragment moved toward the partner telomere after the arm was cut (white line in the first image) but once the tether was cut (white line in the fourth image) the arm fragment stopped moving and drifted away. The black line in the first image represents $5 \mu \mathrm{m}$.

Figure 3: an arm fragment moved back to the arm stub when its tether was cut in a PtK cell. The arm fragment (indicated by a white arrow) moved toward the other telomere after the arm was cut (white line in the second image) and when its tether was cut (white line in the fourth image) the arm fragment moved back to its original arm. The black line in the first image represents $5 \mu \mathrm{m}$.

Figure 4: arms shrink when their tethers are cut in a PtK cell. After their tether was cut (white line in the second image) both chromosome arms shortened. The white arrows indicate the two telomeres. The black line in the first image represents $10 \mu \mathrm{m}$.

Figure 5: the percentage shortening of chromosomes arms when tethers are cut in PtK cells is independent of initial arm length.

Figure 6: the percentage shortening of chromosomes arm stubs when tethers are cut in PtK cells is independent of initial stub length.

Figure 7: not all arm fragments move in PtK cells even at the same tether length. Figure 7A: only one of the two arm fragments (indicated by white arrows) formed when the two arms were cut (white line in the first image) moved toward the opposite telomere. The black line in the first image represents $5 \mu \mathrm{m}$. Time-lapsed images of these (and other cuts) in this cell are presented in the 
Supplemental video. Figure 7B: one of the four arm fragments (white arrows) formed when the arms were cut (white line in the second image) does not move toward the partner telomere. None of the three that moved reached the other telomeres. The black line in the first image represents $5 \mu \mathrm{m}$.

Figure 8: arm fragments in PtK cells tend to move more slowly when their tethers are longer.

Figure 9: In PtK cells, arm fragments with longer tethers are less likely to move toward the opposite telomere, or to move across the equator.

Figure 10: in PtK cells, the longer the tether the less likely that the arm fragment will move.

Figure 11: in PtK cells chromosome arms shorten the by same fractional amount at all tether lengths after their tethers are cut.

Figure 12: in PtK cells, chromosome arms shorten (when tethers are cut) at all tether lengths.

Figure 13: Arm fragments in different cell types move toward partner telomeres. Figure 13A: After arms in an anaphase flatworm (Mesostoma) spermatocyte were cut (black line in the second frame) the arm fragment (indicated by a white arrow) moved across the equator to its partner telomere. Figure 13B: after an arm in a prometaphase Mesostoma spermatocyte (Ferraro-Gideon et al., 2014, describe this unusual cell division) was cut (white line in the first image), the arm fragment (indicated by a white arrow) moved across the equator to the partner telomere. Figure 13C: after an arm in an anaphase cricket spermatocyte was cut (white line, second image) the resultant arm fragment (indicated by a white arrow) moved toward its partner telomere. When its tether was cut (white line in the third and fourth images) the arm fragment stopped moving and the arm of the partner shortened in length. Figure 13D: after an arm in an anaphase cellar spider spermatocyte was cut (white line, first and second images) the arm fragment (indicated by a white arrow) moved to the partner telomere. Figure 13E: after an arm in an anaphase black widow spider spermatocyte was cut (white line, first image), the arm fragment (white arrow) moved backwards toward the partner telomere. Figure 13F: after an arm in an anaphase U2OS cell was cut (white line, second image), the resultant arm fragment (indicated by a white arrow) moved backwards toward its partner telomere. The black lines in the first images in $\mathbf{A}$ and $\mathbf{B}$ represent $10 \mu \mathrm{m}$; those in $\mathbf{C}, \mathbf{D}, \mathbf{E}$ and $\mathbf{F}$ represent $5 \mu \mathrm{m}$. 


\section{SUPPLEMENTARY VIDEO}

The supplementary video is of the cell illustrated in Figure 7A. It shows the laser cuts illustrated in that figure and shows additional cuts as well. The cuts are of arms at the red line at 13:40:28; of arms at the green line at 13:41:09; of arms at the red lines at 13:43:13; of a tether at the red line at 13:42:52; and of an arm at the red line at 13:46:39. 


\section{TABLES}

TABLE 1: comparison of PtK cell arm fragment and anaphase chromosome velocities

\begin{tabular}{|l|l|l|}
\hline Arm fragment velocities & $1.8 \mu \mathrm{m} / \mathrm{min} \pm 1.58 \mu \mathrm{m} / \mathrm{min}(\mathrm{SD})$ & $\mathrm{N}=50$ \\
\hline Anaphase chromosome velocities & $0.4 \mu \mathrm{m} / \mathrm{min} \pm 0.29 \mu \mathrm{m} / \mathrm{min}(\mathrm{SD}$, & $\mathrm{N}=37$ \\
\hline
\end{tabular}

TABLE 2: Shortening of chromosome arms after cutting their tethers

Final length/initial length

\begin{tabular}{|l|l|l|}
\hline PtK cell chromosome arms & $0.89 \pm 0.025(\mathrm{SD})$ & $\mathrm{N}=31$ \\
\hline PtK cell chromosome stubs & $0.85 \pm 0.06$ & $\mathrm{~N}=10$ \\
\hline Crane-fly spermatocyte chromosome arms & $0.90 \pm 0.047$ & $\mathrm{~N}=24$ \\
\hline
\end{tabular}

\section{TABLE 3: Velocities of arm fragments in different cells}

CELL Range of velocities number measured

\begin{tabular}{|c|c|c|}
\hline PtK cells & $0.5-6.2 \mu \mathrm{m} / \mathrm{min}$ & 50 \\
\hline crane-fly $($ Nephrotoma $)$ spermatocytes ${ }^{1}$ & $1-22 \mu \mathrm{m} / \mathrm{min}$ & 37 \\
\hline crane-fly (Nephrotoma) spermatocytes ${ }^{2}$ & $1-18 \mu \mathrm{m} / \mathrm{min}$ & 13 \\
\hline Cricket (Acheta) spermatocytes & $1-11 \mu \mathrm{m} / \mathrm{min}$ & 5 \\
\hline Cellar spider (Pholcus) spermatocytes & $1-7 \mu \mathrm{m} / \mathrm{min}$ & 4 \\
\hline Black widow spider (Latrodectus) spermatocytes & $5-7 \mu \mathrm{m} / \mathrm{min}$ & 2 \\
\hline Flatworm (Mesostoma) spermatocytes & $7-13 \mu \mathrm{m} / \mathrm{min}$ & 2 \\
\hline U2OS (human) cells & $2-13 \mu \mathrm{m} / \mathrm{min}$ & 6 \\
\hline
\end{tabular}

1: data from LaFountain et al., 2002

2: data from Sheykhani et al., 2017 


\section{FIGURE 1}

\section{A}

$02212201513 \mathrm{~h} 33 \pi 07 \mathrm{~s}$

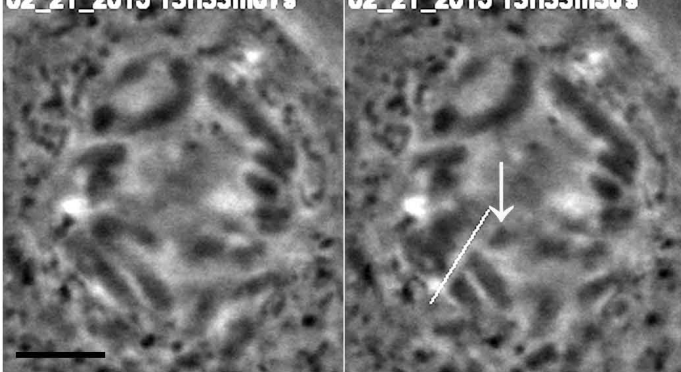

02 21_2015 13h33 $\mu \mathrm{Bg} .68$
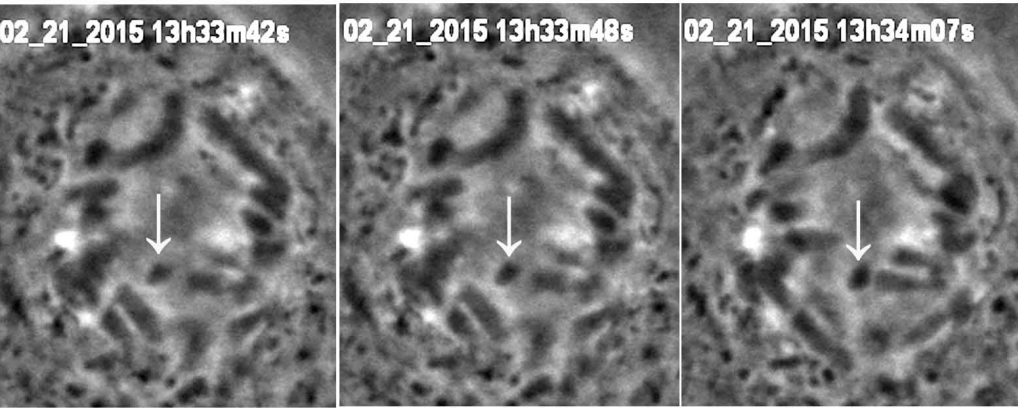

B
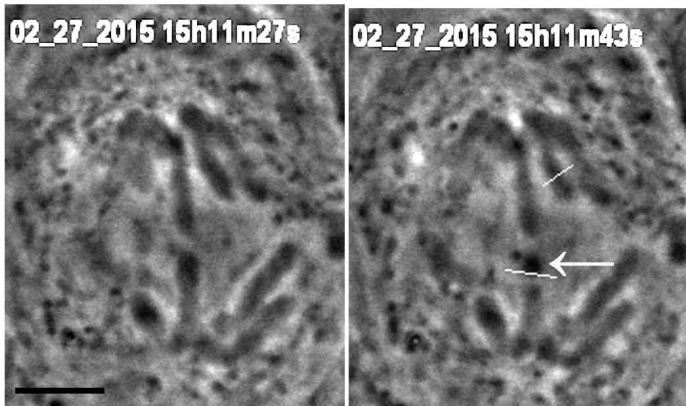

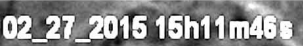

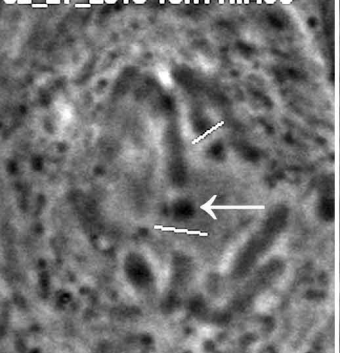

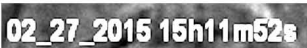

02.27 2015 15hi2mdos
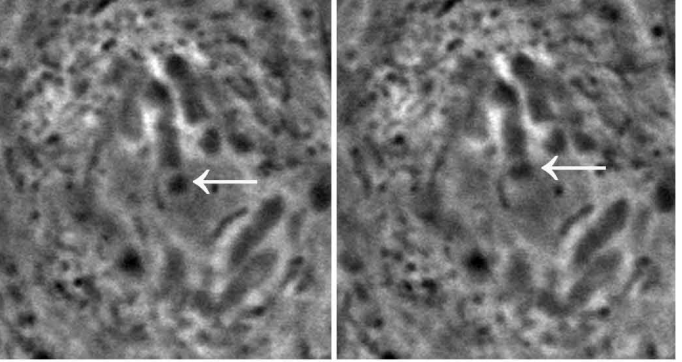


\section{FIGURE 2}

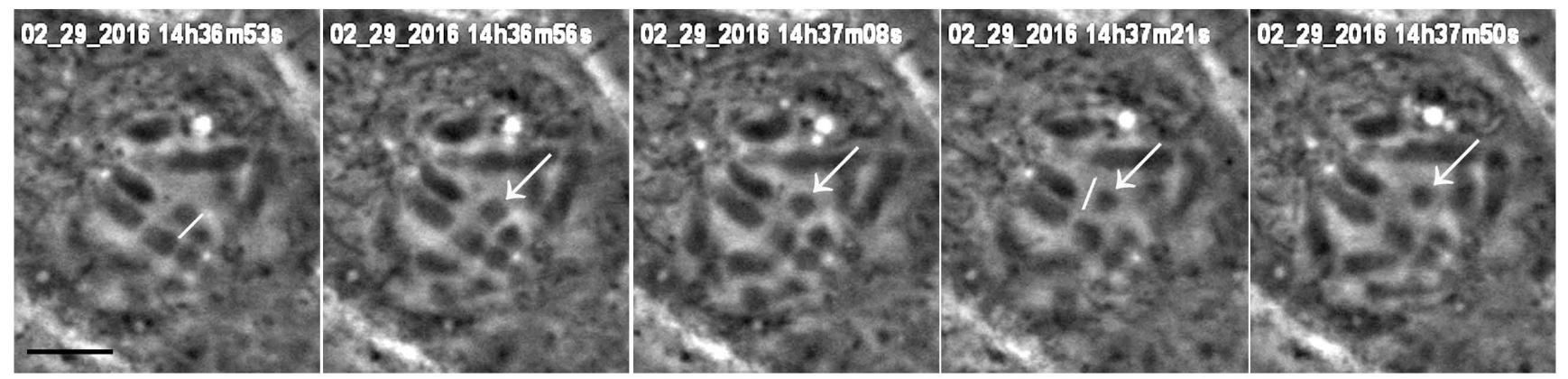




\section{FIGURE 3}

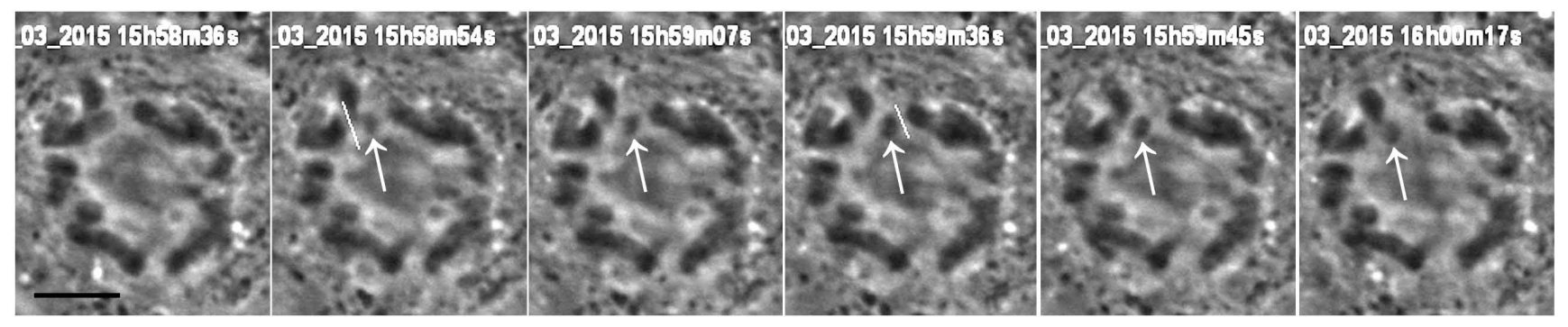




\section{FIGURE 4}

$0213 \quad 2016 \quad 14 h 17 m^{21 s}$

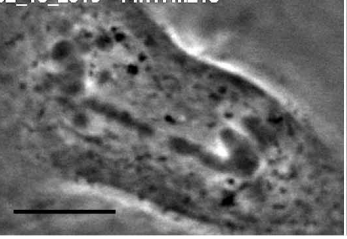

02_13_2016 14h17m24s

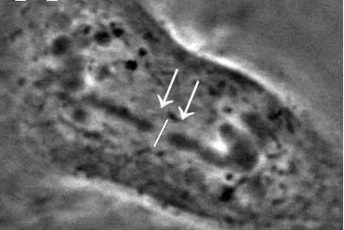

02_13_2016 14h17m46s

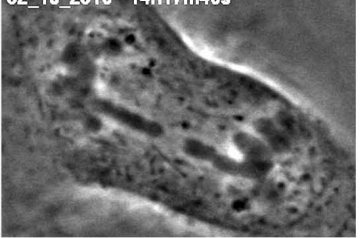

02_13_2016 14h18m59s

02_13_2016 14h18m37s

02_13_2016 14h18m09s
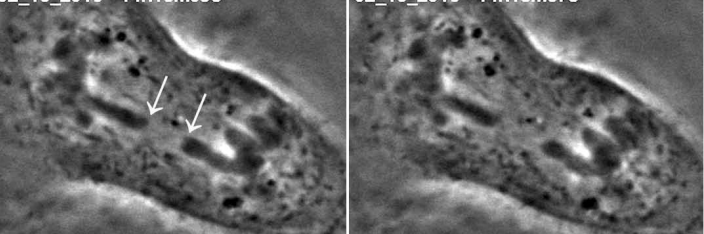

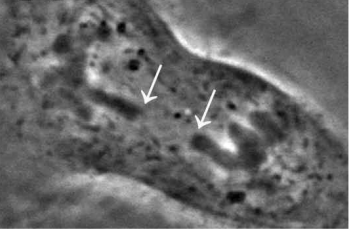




\section{FIGURE 6}

\section{Shortening of arm stubs}

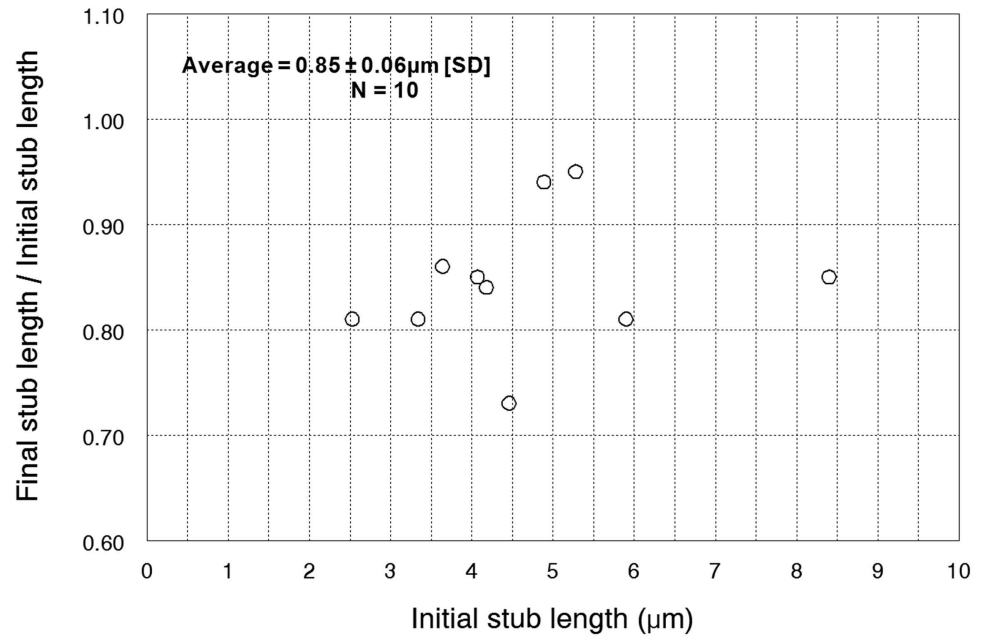




\section{FIGURE 7}

A

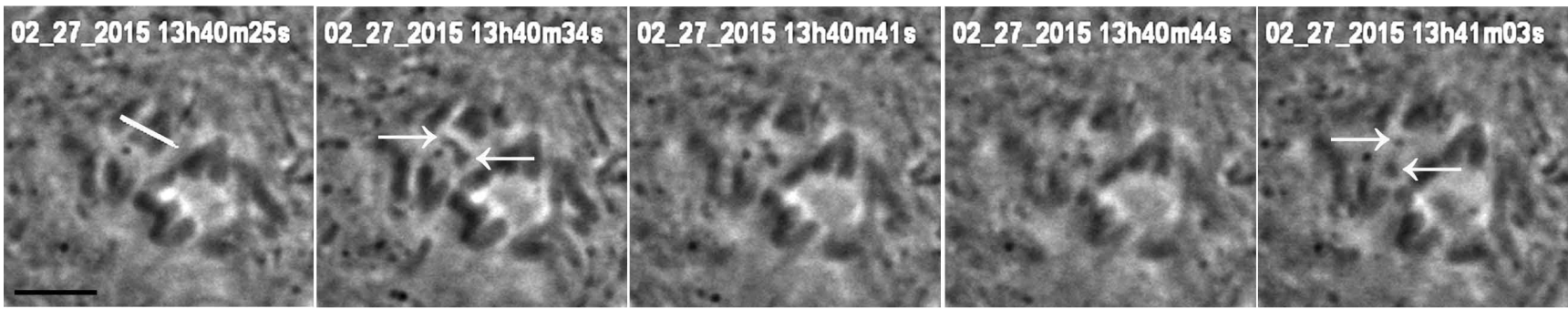

B

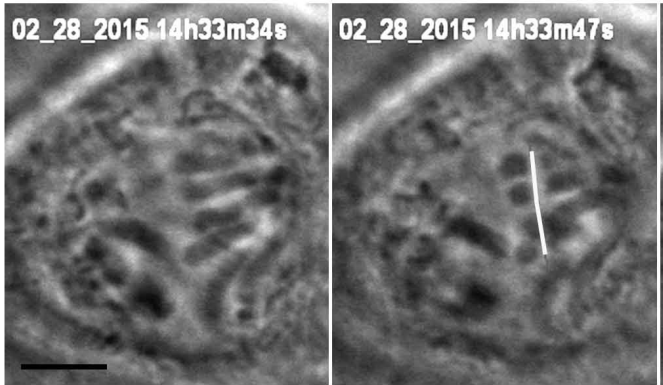

02 28_2015 14h34m00s

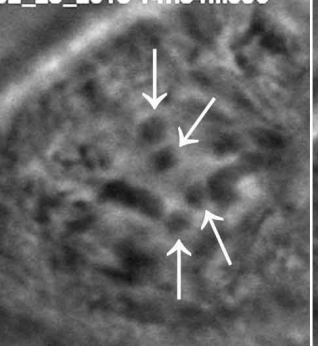

02282015 14h34m31s

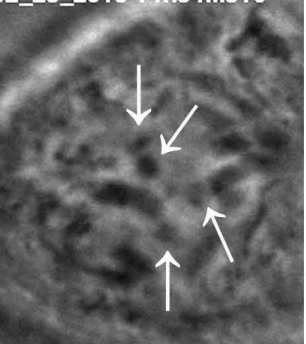

02_28_2015 14h34m35s 


\section{FIGURE 8}

\section{Velocities of arm fragments}

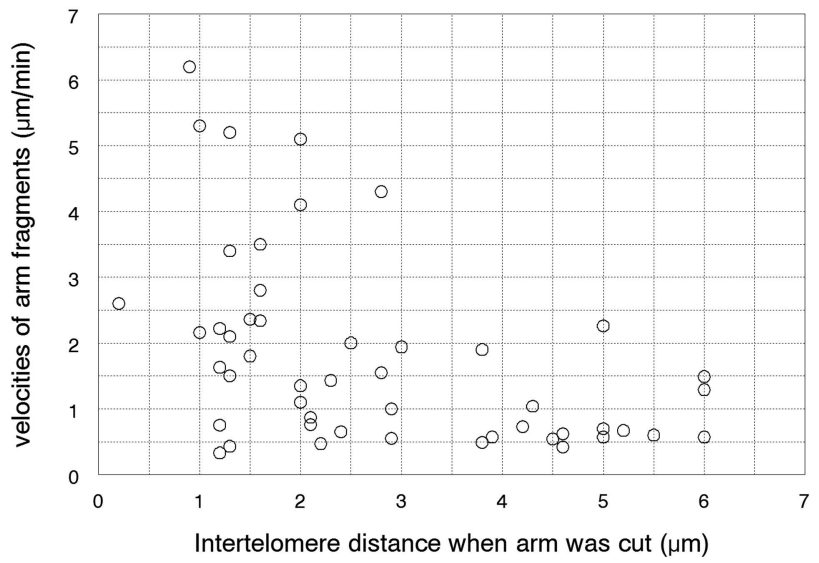




\section{FIGURE 9}

\section{Distances arm fragments move}
- Fragment moves to partner
Moves past equa- tor, not to partner
Does not cross equator

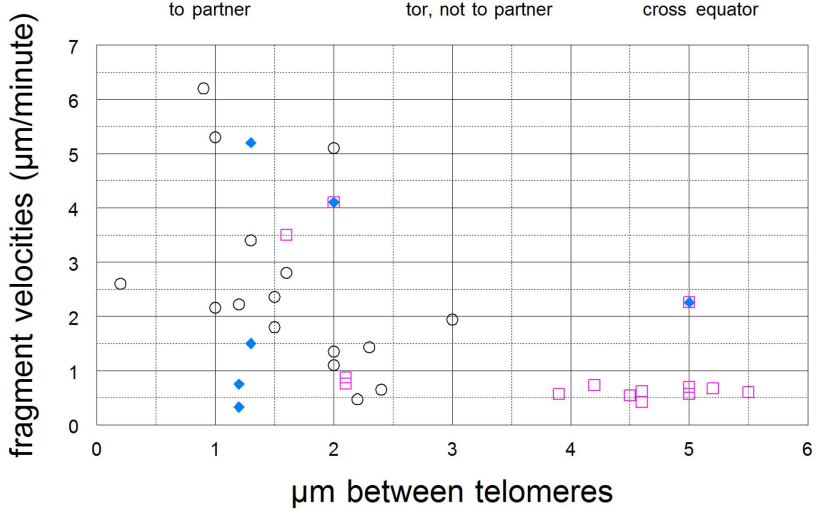




\section{FIGURE 10}

Fragment movement at different tether lengths

$\square$ Fragments moved

Fragments did NOT move

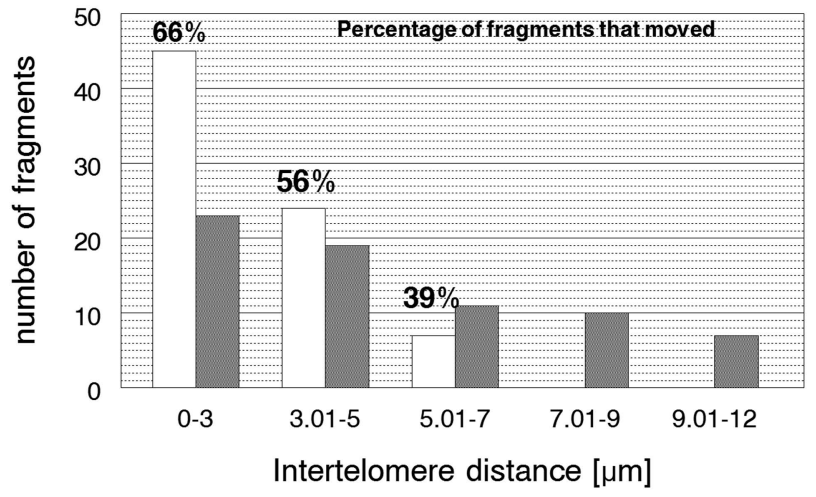


Arm length changes after cuts of different length tethers

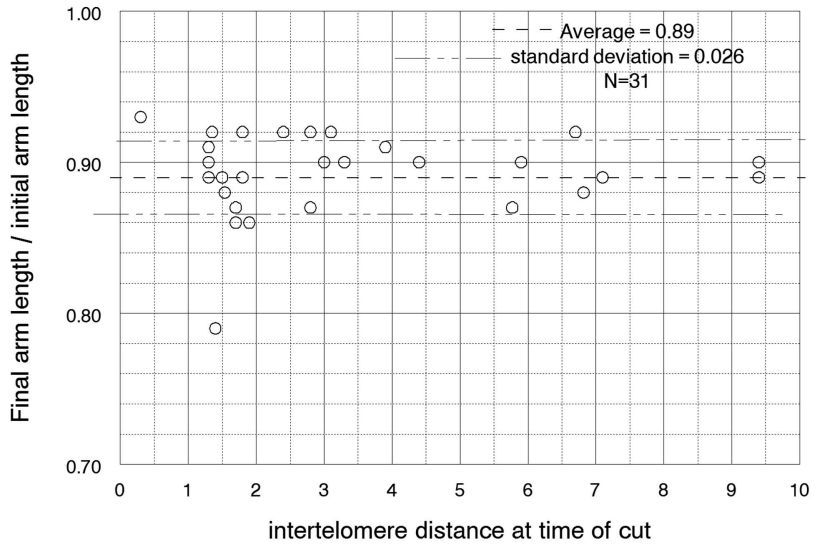




\section{FIGURE 12}

Arm shortening [tether cuts] at different tether lengths

Arms shortened

Arm did NOT shorten

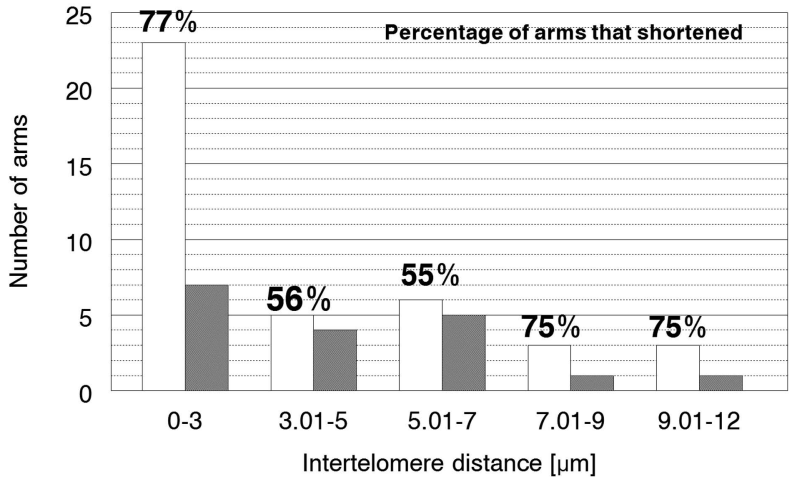




\section{FIGURE 14}

A Mesostoma spermatocytes - cut in anaphase:
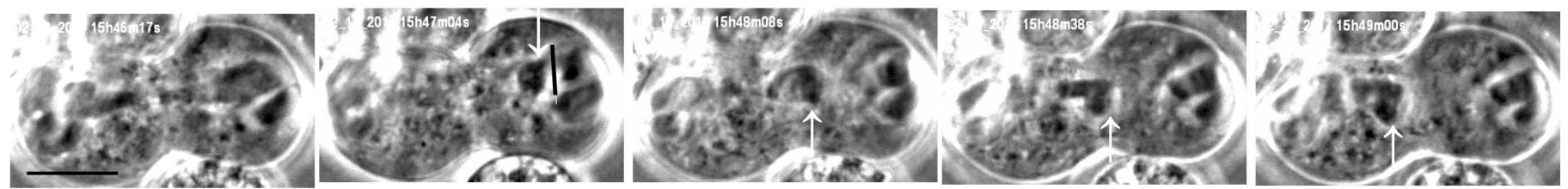

B Mesostoma spermatocytes - cut in prometaphase:
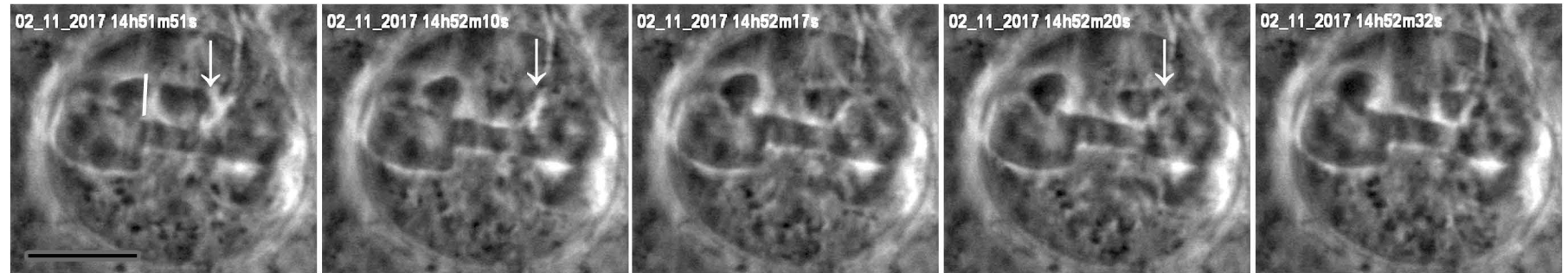

C Acheta spermatocytes meiosis-1:

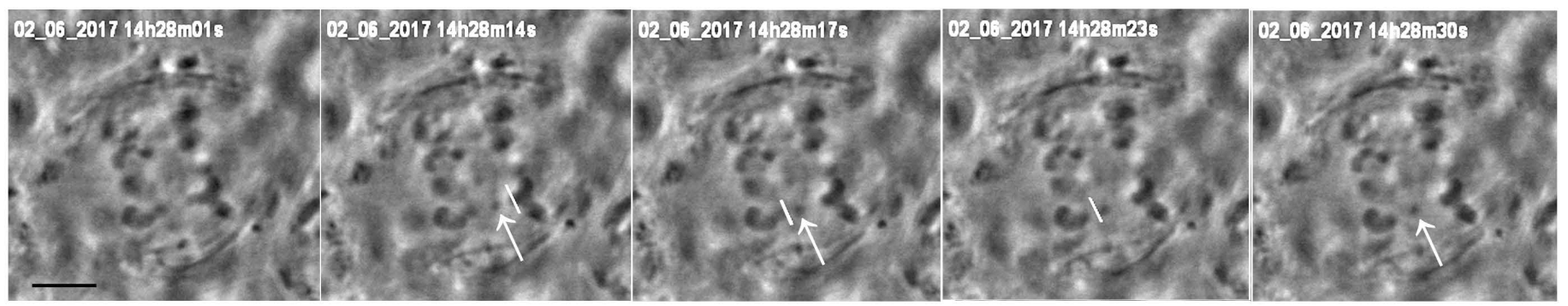

\section{Acheta spermatocytes meiosis-2:}

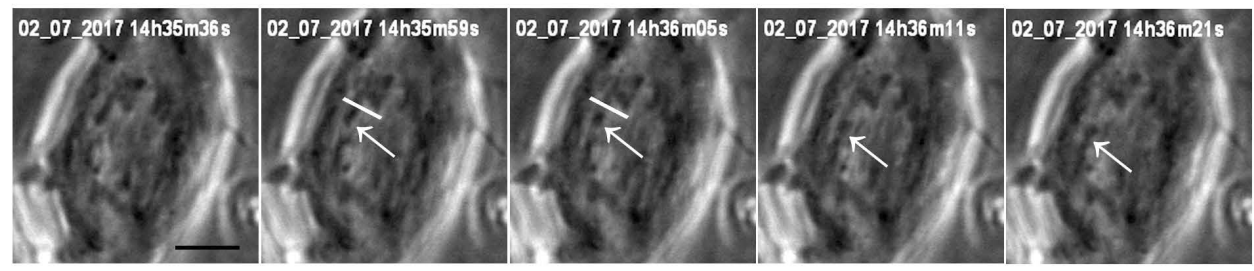

E Cellar spider spermatocytes:

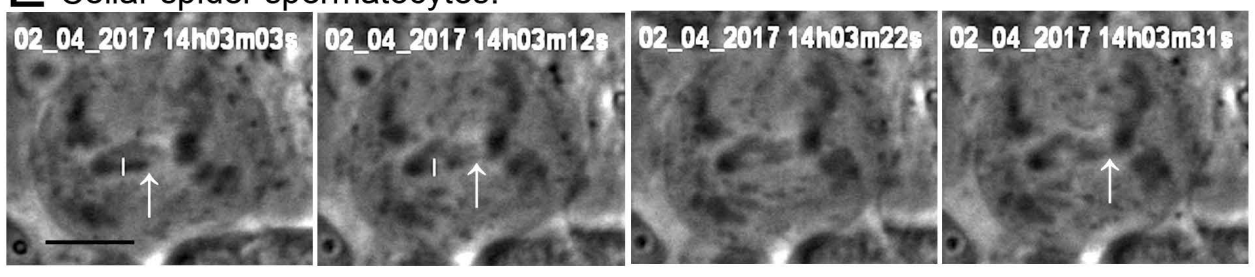

$F$ Black widow spider spermatocytes:

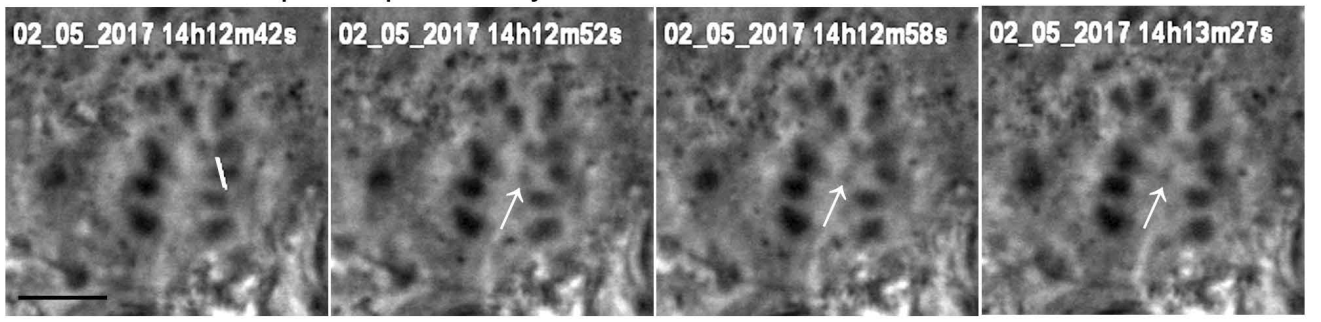

\section{G u2Os cells:}
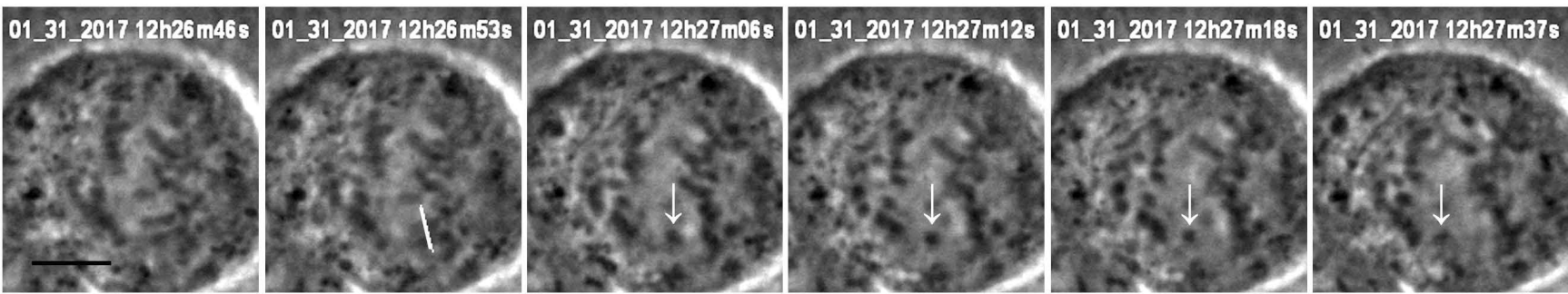